\title{
Home Administration of CVD 103-HgR: A Live Attenuated Oral Cholera Vaccine
}

\author{
R. Paul Duffin, ${ }^{\star}$ Michael Delbuono, Lawrence Chew, James Johnstone, Volker Niedan, Pascal Schwarz, Paul Shabram, \\ and Amish A. Patel \\ Emergent BioSolutions, Gaithersburg, Maryland
}

\begin{abstract}
Vaccination is a well-established means for prevention and spread of disease in people traveling abroad. Although vaccines to diseases such as cholera are recommended by world health agencies, they are seldom required even when traveling to endemic regions. Consequences of noncompliance can affect traveler's health and spread diseases to new regions, as occurred in Haiti in 2010 when United Nations peacekeepers from Nepal, where a cholera outbreak was underway, introduced the disease to the region. Steps to increase vaccine recommendation compliance should therefore be an integral part of vaccine development. PXVX0200 contains Center for Vaccine Development 103HgR live, attenuated recombinant Vibrio cholerae vaccine strain, and is indicated for single-dose immunization against the bacteria that causes cholera. It is supplied as one buffer and one active component packet to be mixed into water and ingested. Administration instructions are designed to be "user friendly" with flexibility for self-administration, thus promoting compliance. Studies to support self-administration were conducted to cover stability of the vaccine outside of normal storage conditions, potency in case of misadministration, and disposal procedures to minimize environmental impact. The principal findings showed that the stability of vaccine was maintained under conditions allowing for transport times and temperature conditions as well as when misadministration errors were made. Finally, the vaccine was effectively neutralized with hot water and soap to prevent bacterial environmental contamination in the event of an accidental spill. The conclusion is that PXVX0200 oral vaccine is stable, easy to formulate and dispose of, and is amenable to selfadministration.
\end{abstract}

\section{INTRODUCTION}

Vaccine compliance for diseases such as cholera that do not generally affect developed countries is uniquely challenging. Cholera is an acute disease that causes severe diarrhea which can kill within hours if left untreated, but vaccine requirements for cholera-endemic regions are rare, leaving it up to the traveler's discretion whether to follow government or nongovernmental organization vaccine recommendations. With proper medical treatment, the mortality rate of cholera is quite low, but untreated cholera can have a mortality rate of more than $50 \%{ }^{1}$ Local political, social, and economic conditions can affect resident's access to treatment and preventative measures. As a result, cholera causes 1.3-4.0 million cases and 21,000-143,000 deaths/year ${ }^{2}$ despite the availability of treatment and safe and effective vaccines. Travelers can unwittingly spread the disease with disastrous consequences, ${ }^{3}$ as in 2010 when Haiti experienced a cholera outbreak despite the absence of the disease in the region since the late 1800s. The sudden appearance of cholera in Haiti was traced to humanitarian aid workers traveling from Nepal where an outbreak of cholera was underway. ${ }^{4}$ Since 2010 , cholera infected more than 800,000 Haitians, causing 9,606 deaths, and there is evidence of established environmental reservoirs of Vibrio cholerae ${ }^{5}$ which could contribute to future epidemics, thus underscoring the importance of strict compliance with health agencies' vaccine recommendations.

The development of an effective, potent cholera vaccine with characteristics that promise to enhance compliance was undertaken by Emergent BioSolutions. Considerations for product development included dosing frequency, ease of preparation and route of administration, product stability, and physicochemical properties that were conducive for self-

*Address correspondence to Ryan Paul Duffin, Emergent BioSolutions, 400 Professional Dr., Suite 400 Gaithersburg, MD20879. E-mail: duffinp@ebsi.com administration and disposal at home. Live attenuated cholera vaccine strain Center for Vaccine Development (CVD) 103$\mathrm{HgR}$, produced from CVD, strain 103, HgR mercury resistant bacterium, is indicated for active immunization against $V$. cholerae, the bacteria that causes cholera. The CVD 103$\mathrm{HgR}$ vaccine strain has been licensed outside of the United States under the trade names Orochol and Mutachol, although production was discontinued in 2001 for business reasons. PaxVax redeveloped the CVD 103-HgR vaccine as PXVX0200 and received market authorization in 2016 through the Food and Drug Administration (FDA) under the trade name Vaxchora $^{\circledR}$, (Emergent Travel Health, Inc., Redwood City, CA) the first cholera vaccine approved in the United States. The first iteration of the PXVX0200 vaccine, stable at $-25^{\circ} \mathrm{C}$ to $-15^{\circ} \mathrm{C}$ (frozen), was not considered for self-administration. The second iteration of PXVX0200, approved by the FDA as well as by the European Medicines Agency (EMA), has several advantages as a stable, single-dose, orally administered vaccine that can be stored in a refrigerator $\left(2-8^{\circ} \mathrm{C}\right)$, and is resistant to freeze/ thaw cycles, which limits logistical complexity and may be more effective during vaccination campaigns. ${ }^{6}$ These attributes have been identified as advantageous by healthcare stakeholders. ${ }^{7}$ Self-administration, shown to improve compliance and reduce vaccine cost, ${ }^{8,9}$ was therefore considered during the final steps in the development of the PXVX0200 vaccine. To support selfadministration, the stability of the vaccine was assessed under conditions outside of current prescribing parameters, and was further characterized to provide confidence in the patient's ability to transport the vaccine, prepare it using only package leaflet $(\mathrm{PL})$ instructions, self-administer, and dispose of the remnants safely.

Stability of PXVX0200 vaccine was characterized at temperatures up to $32^{\circ} \mathrm{C}$ for defined time periods as these conditions could be expected during transport from the pharmacy to the patient's home. Misadministration scenarios were tested to show that the vaccine is robust and retains potency, even when mistakes are made in preparing the vaccine. We 
therefore conducted studies to characterize properties of PXVX0200 product under these conditions to ensure that potency and stability of the vaccine could be maintained for safe and effective self-administration. Results confirmed that PXVX0200 vaccine potency is maintained under transport and self-administration conditions and can be safely and effectively self-administered even when common misadministration errors occur.

\section{MATERIALS AND METHODS}

PXVX0200 live oral cholera vaccine contains a buffer and a vaccine (active) component that is supplied as two separate packets (sachets) that are reconstituted in water and administered orally. As stated in the PL, the vaccine is "indicated for active immunization against disease caused by $V$. cholerae," with a lower potency limit of $4 \times 10^{8}$ colony-forming unit (CFU)/ dose. Live vaccines are, by nature, less stable than killed antigen vaccines but can produce a long-lasting immune response with a single dose. ${ }^{10}$ For self-administration of a live vaccine to be effective, stability outside of normal storage conditions must be characterized. Two lots of PXVX0200 active vaccine component (live and attenuated $V$. cholerae CVD $103-\mathrm{HgR}$ ), supplied as "sachet 2" in the product package, were chosen to characterize early- and late-phase decay trends for stability. The first lot (Lot 076) was produced before commercialization as part of the validation process and was the oldest available at the time of the study. Vaccine component 076 was paired with a buffer component packet (sachet 1 in the PL). The second vaccine component lot (Lot 277) included vaccine and buffer component packets from the most recent commercially available product.

All stability time points were determined using product reconstituted in chilled buffered peptone water (Hardy Diagnostics) per the standard operating procedure (SOP) for the PXVX0200 vaccine stability program. All misadministration experiments used non-carbonated, Aquafina brand purified bottled drinking water, except where noted.

PXVX0200 active component potency was defined as $4 \times 10^{8}$ to $2 \times 10^{9}$ CFUs of CVD 103-HgR per dose of vaccine component, based on experience with previous commercial formulations of CVD 103-HgR and challenge studies performed in the United States. ${ }^{11,12}$ One dose of PXVX0200 vaccine consists of $4.5 \mathrm{~g}$ of buffer and $2 \mathrm{~g}$ of active components. In all cases, 100 $\mu \mathrm{L}$ of reconstituted vaccine sample was diluted in chilled buffered peptone water to achieve concentrations within the linear range of the CFU assay. Samples were plated onto tryptic soy agar (TSA) plates (BioMerieux, Lombard, IL) using the WASP Touch Spiral Plater (Don Whitley Scientific, Shipley, United Kingdom) as per the relevant SOP. Colonies were quantified using the ProtoCOL 3 Plus automatic colony counting system (Synbiosis, Frederick, MD).

Three lots of vaccine component (lots 230, 231, and 232), produced as part of the validation process, were used for longterm stability studies at $25^{\circ} \mathrm{C} / 24$ hours. Samples were plated on fresh TSA plates made onsite in Bern, Switzerland, using the EasySpiral Dilute system (Interscience, Saint Nom, France). Colonies were counted manually.

Stability of PXVX0200 vaccine outside of prescribed temperature storage. An important consideration is the time that the vaccine is unrefrigerated during transport from the pharmacy to the patient's home. Typically, this information is provided in a $25^{\circ} \mathrm{C}$ stability statement within the storage section of the PL or summary of product characteristics (SmPC) that includes the conditions and shelf-life (when the product should be discarded). However, it is also prudent to study the stability at higher temperatures that can occur in many regions of the world.

Three stability determinations, under different temperature and humidity conditions, were used for PXVX0200 vaccine self-administration stability studies: $25^{\circ} \mathrm{C} / 60 \%$ relative humidity $(\mathrm{RH}), 30^{\circ} \mathrm{C} / 65 \% \mathrm{RH}$, and $32^{\circ} \mathrm{C} / 65 \% \mathrm{RH}$. Twenty-five and $30^{\circ} \mathrm{C}$ are typical conditions suggested in the U.S. FDA and European Medicines Agency (EMA) guidelines as part of the stability program, ${ }^{13,14}$ and $32^{\circ} \mathrm{C}$ correlates to the hottest average month in Europe (July) for the cities with the highest temperatures (Athens, Greece, Podgorica, Montenegro, Rome, Italy, Tirana, and Albania) according to their respective national meteorological agencies. ${ }^{15}$

The stability of PXVX0200 active component following short-term and longer term temperature excursion scenarios was tested. The aim of short-term stability experiments was to characterize the vaccine component under conditions encountered when the vaccine is transported from the pharmacy to the patient's home, whereas the longer-term stability experiments aimed to determine the effects of multiple temperature excursions from $2-8^{\circ} \mathrm{C}$ to $25^{\circ} \mathrm{C}$ on stability.

Short-term stability of PXVX0200 vaccine at 25,30 , and $32^{\circ} \mathbf{C}$. Individual vaccine component packets from lots 076 and 277 with their respective buffer component packets were removed from $2^{\circ} \mathrm{C}$ to $8^{\circ} \mathrm{C}$ storage conditions and placed at $30^{\circ} \mathrm{C} / 65 \% \mathrm{RH}$ for up to 24 hours, or $32^{\circ} \mathrm{C} / 65 \% \mathrm{RH}$ for up to 48 hours. Stability data at $25^{\circ} \mathrm{C}$ were generated for lots 230,231 , and 232 , as shown in the following section entitled "long-term stability of vaccine following $25^{\circ} \mathrm{C} / 24$-hour excursions." To better characterize the short-term stability beyond 24 hours, lot 076 vaccine and respective buffer component packets were removed from the refrigerator and placed at $25^{\circ} \mathrm{C} / 60 \%$ $\mathrm{RH}$ for up to 7 days. As there was no expectation to exceed 24 hours at $25^{\circ} \mathrm{C}$ in the PXVX0200 SmPC, lot 277 was not tested at this condition.

In all cases, packets were taken at specific time points and reconstituted in chilled buffered peptone water, diluted, and plated.

Long-term stability of vaccine following $25^{\circ} \mathrm{C} / 24$-hour excursions. PXVX0200 active component packets from lots 230,231 , and 232 with respective buffer component packets were removed from $2^{\circ} \mathrm{C}$ to $8^{\circ} \mathrm{C}$ storage, placed at $25^{\circ} \mathrm{C}$ for 12 or 24 hours, and returned to the refrigerator until testing. Samples were also cycled between $25^{\circ} \mathrm{C}$ and $2-8^{\circ} \mathrm{C}$ three times to determine the effects of multiple excursions. These packets were returned to $2-8^{\circ} \mathrm{C}$ storage for at least 24 hours between cycles. At the end of the cycle excursions, all packets were returned to $2-8^{\circ} \mathrm{C}$. Product potency (CFU/dose) data, determined from samples subjected to the various aforementioned conditions (or scenarios), was compared with data from long-term stability studies at $2-8^{\circ} \mathrm{C}$ using the same lot of vaccine and buffer component packets without cycling.

PXVX0200 vaccine preparation and misadministration. PXVX0200 vaccine PL instructions for preparation and administration (Supplemental Figure 1) are straightforward and easy to follow; however, mistakes can be made, resulting in misadministration. During the development of the PXVX0200 vaccine $P L$, we investigated several scenarios where mistakes 
were most likely to occur during self-preparation and administration at home (Figure 1).

PXVX0200 vaccine is supplied as a separate buffer (sachet 1) and active component powder packets (sachet 2) that are emptied stepwise into $100 \mathrm{~mL}$ of water, mixed, and consumed. It is essential that the buffer is reconstituted first in $100 \mathrm{~mL}$ of bottled water, followed by addition of the vaccine component. Detailed visual and written instructions for patients on how to take Vaxchora CVD 103-HgR vaccine are included in the SmPCs PL section B.3 entitled "How to take Vaxchora" (Supplement 1).

Stability of PXVX0200 vaccine: Misadministration scenarios. Studies were performed to measure the potency of PXVX0200 product when not prepared as per the PL reconstitution instructions.

Stability of reconstituted PXVX0200 vaccine. The PL instructs to drink the vaccine in no more than 15 minutes after reconstitution. To determine the effect on the vaccine if reconstituted for longer than 15 minutes, vaccine component lots 076 and 277 and respective buffer packet lot contents were reconstituted at room temperature in non-carbonated bottled water (Aquafina) as per the PL reconstitution instructions. Samples were taken at $0.5,1,2$, and 4 hours and tested for potency (CFU/dose).

Incorrect water volume for reconstitution. The PXVX0200 vaccine $P L$ instructs that the buffer and active components are reconstituted in $100 \mathrm{~mL}$ of water. A study was performed to assess the potency of the vaccine reconstituted if it is mistakenly reconstituted in a different volume of water. PXVX0200 vaccine lot 076 and lot 277 were reconstituted in 50, 100, or $150 \mathrm{~mL}$ at room temperature in Aquafina bottled water. Samples were taken for potency measurements immediately following reconstitution of the vaccine component as per the $\mathrm{PL}$ instructions, and again at 15 and 30 minutes postreconstitution. Buffer and active vaccine components reconstituted together, or in reverse order. The PL instructs that the buffer component (sachet 1) is to be added to the water first, stirred until it is completely dissolved, and then active component (sachet 2) is to be added to the buffered water and stirred for 30 seconds. We determined the effects of two misadministration errors on potency of the active component.

Packets reconstituted together. PXVX0200 buffer and vaccine component packets were removed from $2^{\circ} \mathrm{C}$ to $8^{\circ} \mathrm{C}$ storage and opened with scissors by holding the packets together and cutting across the top of both packets, taking precautions to not touch contents. The contents were then emptied simultaneously into $100 \mathrm{~mL}$ of premeasured, room temperature Aquafina bottled water, and then stirred for 30 seconds. Samples were taken for potency measurements immediately, and at 15 and 30 minutes after reconstitution.

Packets reconstituted in reverse order. PXVX0200 buffer and vaccine component packets were removed from $2^{\circ} \mathrm{C}$ to $8^{\circ} \mathrm{C}$ storage, and the vaccine component packet was emptied into $100 \mathrm{~mL}$ of unbuffered, Aquafina bottled water at room temperature and stirred for 30 seconds. Then, the buffer component packet was emptied into $100 \mathrm{~mL}$ of water with reconstituted vaccine component, and samples were taken for potency measurements immediately following reconstitution, and at 15 and 30 minutes post-reconstitution.

Safe disposal of reconstituted PXVX0200 product. The active ingredient of PXVX0200 vaccine is CVD 103-HgR, a genetically modified live $V$. cholerae organism. Genetically modified organisms (GMOs) should be limited in their environmental dispersal, so studies were performed to test the effect of different decontamination procedures (e.g., soap and water at different temperatures) on PXVX0200 product potency.

Handwashing with soap and water has been shown to be an effective method for reducing the occurrence of enteric diseases including cholera. ${ }^{16}$ Hot water alone and hot water with soap were tested as means to effectively neutralize $V$. cholerae CVD 103-HgR. The temperature of "hot water" for these experiments was defined by the European Child Safety Alliance, which recommends that water exiting the taps does not exceed $50^{\circ} \mathrm{C}$ to avoid water scalds. ${ }^{17}$ Two methods of inactivating CVD 103-HgR were tested:

Hot water only, worst-case scenario. A full dose of PXVX0200 vaccine was reconstituted in $100 \mathrm{~mL}$ of Aquafina bottled water, preheated to $40^{\circ} \mathrm{C}, 50^{\circ} \mathrm{C}$, and $60^{\circ} \mathrm{C}$. Samples were taken for

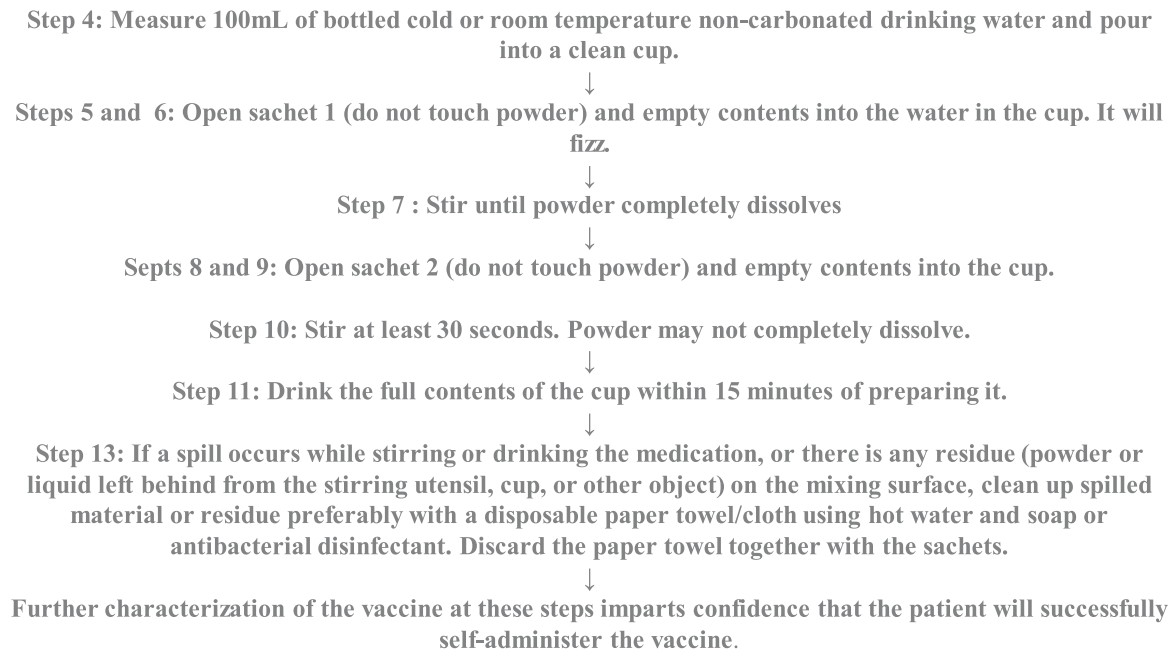

FIGURE 1. Steps from PXVX0200 package leaflet where misadministration errors are most likely to occur. 
potency measurements immediately following and at 15 minutes post-reconstitution.

The temperature of the vaccine reconstituted in preheated water was allowed to cool at ambient temperature over the course of the experiment. This represents the worst-case scenario where the maximum expected amount and concentration of PXVX0200 product require neutralization (i.e., a full dose of PXVX0200 vaccine).

Residual vaccine. To simulate residual amounts of active component remaining following reconstitution, PXVX0200 vaccine was reconstituted at room temperature in Aquafina bottled water, and $0.5 \mathrm{~mL}, 1 \mathrm{~mL}$, or $5 \mathrm{~mL}$ were removed and added to $50 \mathrm{~mL}$ of Aquafina water heated to $40^{\circ} \mathrm{C}, 50^{\circ} \mathrm{C}$, and $60^{\circ} \mathrm{C}$. Samples were taken for potency measurements immediately following and at 15 minutes post-reconstitution.

Soap and water: Ambient temperature water and soap. A full dose of PXVX0200 vaccine was reconstituted in $100 \mathrm{~mL}$ of room temperature Aquafina water, and prepared as per the $\mathrm{PL}$ instructions. A volume of $0.1 \mathrm{~mL}$ or $5.0 \mathrm{~mL}$ of Palmolive antibacterial dish liquid was then added to the reconstituted vaccine and stirred vigorously for 5 seconds. Samples were taken for potency measurements immediately following reconstitution, and at 15 minutes and 30 minutes postreconstitution.

Hot water and soap. A full dose of PXVX0200 vaccine was reconstituted in $100 \mathrm{~mL}$ of Aquafina water heated to $50^{\circ} \mathrm{C}$. A volume of $0.1 \mathrm{~mL}$ or $5 \mathrm{~mL}$ of Palmolive antibacterial dish liquid was then added to the reconstituted vaccine, and then stirred vigorously for 5 seconds. Samples were taken for potency measurements immediately following reconstitution, and at 15 minutes post-reconstitution. In all cases, samples taken for potency measurements were immediately diluted into chilled buffered peptone water and plated onto the TSA plates.

\section{RESULTS}

Stability of PXVX0200 vaccine outside of prescribed temperature storage. The stability of PXVX0200 vaccine following short-term and longer-term temperature excursion scenarios was tested to characterize the active component under conditions encountered when the vaccine is transported from the pharmacy to the patient's home (short-term excursion), and to determine the effects of multiple temperature excursions from $2-8^{\circ} \mathrm{C}$ to $25^{\circ} \mathrm{C}$ on (longer term) stability.

Stability data showed that the vaccine and buffer component packet contents were stable under short-term temperature excursion conditions for up to 5 days at $25^{\circ} \mathrm{C}$, up to 12 hours at $30^{\circ} \mathrm{C}$, and up to 6 hours at $32^{\circ} \mathrm{C}$ (Figure $2 \mathrm{~A}-\mathrm{C}$, respectively). However, vaccine potency (CFU/dose) decreased with increases in temperature, and titers were maintained within the acceptable range for the times indicated.

The potency specification for PXVX0200 active component is $4 \times 10^{8}$ to $2 \times 10^{9} \mathrm{CFU} /$ dose. (A) The age of vaccine lot 076 was 5 months for the $25^{\circ} \mathrm{C}$ study. (B) The age of vaccine lot 076 was 8 months and the age of vaccine lot 277 was 5 months for the $30^{\circ} \mathrm{C}$ study. (C) The age of vaccine lot 076 was 8 months and the age of vaccine lot 277 was 5 months for the $32^{\circ} \mathrm{C}$ study.

Vaccine stability temperature excursion studies showed that vaccine potency was maintained following single or multiple temperature excursions from storage at $2-8^{\circ} \mathrm{C}$ storage to $25^{\circ} \mathrm{C}$ (Figure $3 \mathrm{~A}-\mathrm{C}$ ).
Vaccine and buffer component packets were moved from $2-8^{\circ} \mathrm{C}$ storage to $25^{\circ} \mathrm{C}$ storage and back to $2-8^{\circ} \mathrm{C}$, where they were stored until testing. The excursion schedules were as follows: three 8 -hour cycles ( $3 \times 8$ hours), one 12 -hour cycle ( $1 \times 12$ hours), or one 24 -hour cycle $(1 \times 24$ hours). The lots used ranged from 5 to 6 months of age at the time of the study. (A) Vaccine lot 230, (B) vaccine lot 231, and (C) vaccine lot 232.

Vaccine potency (CFU/dose) was retained within the acceptable range following temperature excursions on vaccine that had been stored at $2-8^{\circ} \mathrm{C}$ for up to 18 months. Furthermore, vaccine stability was maintained when cycled three times between $2^{\circ} \mathrm{C}$ and $8^{\circ} \mathrm{C}$ and $25^{\circ} \mathrm{C}$ for a cumulative total of 24 hours.

Stability of PXVX0200 vaccine: Misadministration scenarios. Studies were performed to measure the potency of PXVX0200 active component when not prepared as per the PL reconstitution instructions. A number of scenarios are presented in the in the following sections including deviations from the recommended time of administration (e.g., $\leq 15$ minutes after reconstitution), recommended dilution volumes, and stepwise order for buffer and vaccine preparation were simulated to determine effects on vaccine potency (Figure $4 \mathrm{~A}-\mathrm{C}$ ).

The potency specification for PXVX0200 active component is $4 \times 10^{8}$ to $2 \times 10^{9} \mathrm{CFU} /$ dose. The age of vaccine lot 076 was 8 months for the reconstituted vaccine study. The age of vaccine lot 277 was 5 months for the incorrect reconstitution volume and incorrect packet reconstitution sequence studies. (A) Potency was assessed immediately after reconstitution, and 30 minutes and 1, 2, and 4 hours post-reconstitution. (B) Potency was assessed immediately following reconstitution, and at 15 and 30 minutes post-reconstitution. (C) Potency was assessed under conditions of incorrect reconstitution sequences.

Deviations from recommended time of administration. The current recommendation in the PL instructs patients to drink reconstituted vaccine no more than 15 minutes after reconstitution; however, deviations can occur with selfadministration, especially in the home. Potency data determined at times ranging from 30 minutes up to 4 hours after reconstitution in Aquafina bottled water (room temperature) showed that potency declined over time but was retained above the acceptable lower limit for at least 4 hours (Figure 4A).

Incorrect water volume for reconstitution. The recommended water volume for dilution of buffer and vaccine is $100 \mathrm{~mL}$ of bottled cold or room temperature non-carbonated drinking water. When volumes were adjusted to 50 and $150 \mathrm{~mL}$, vaccine potency was retained above the lower limit for at least 30 minutes post-reconstitution (Figure 4B). This is important because if the patient inadvertently mismeasures the volume of water before reconstitution, the full dose of vaccine will still be administered.

Buffer and active vaccine components reconstituted together, or in reverse order. Instructions state that buffer and vaccine are to be added to water in a stepwise manner with addition of buffer first, followed by vaccine. Misadministration scenarios were tested to determine effects of deviations from this guidance on vaccine potency, including simultaneous reconstitution and reverse order addition of packets.

Packets reconstituted together. Simultaneous addition of buffer and vaccine component packet contents had no effect on vaccine potency compared with vaccine that was prepared in the correct order (Figure 4C). 

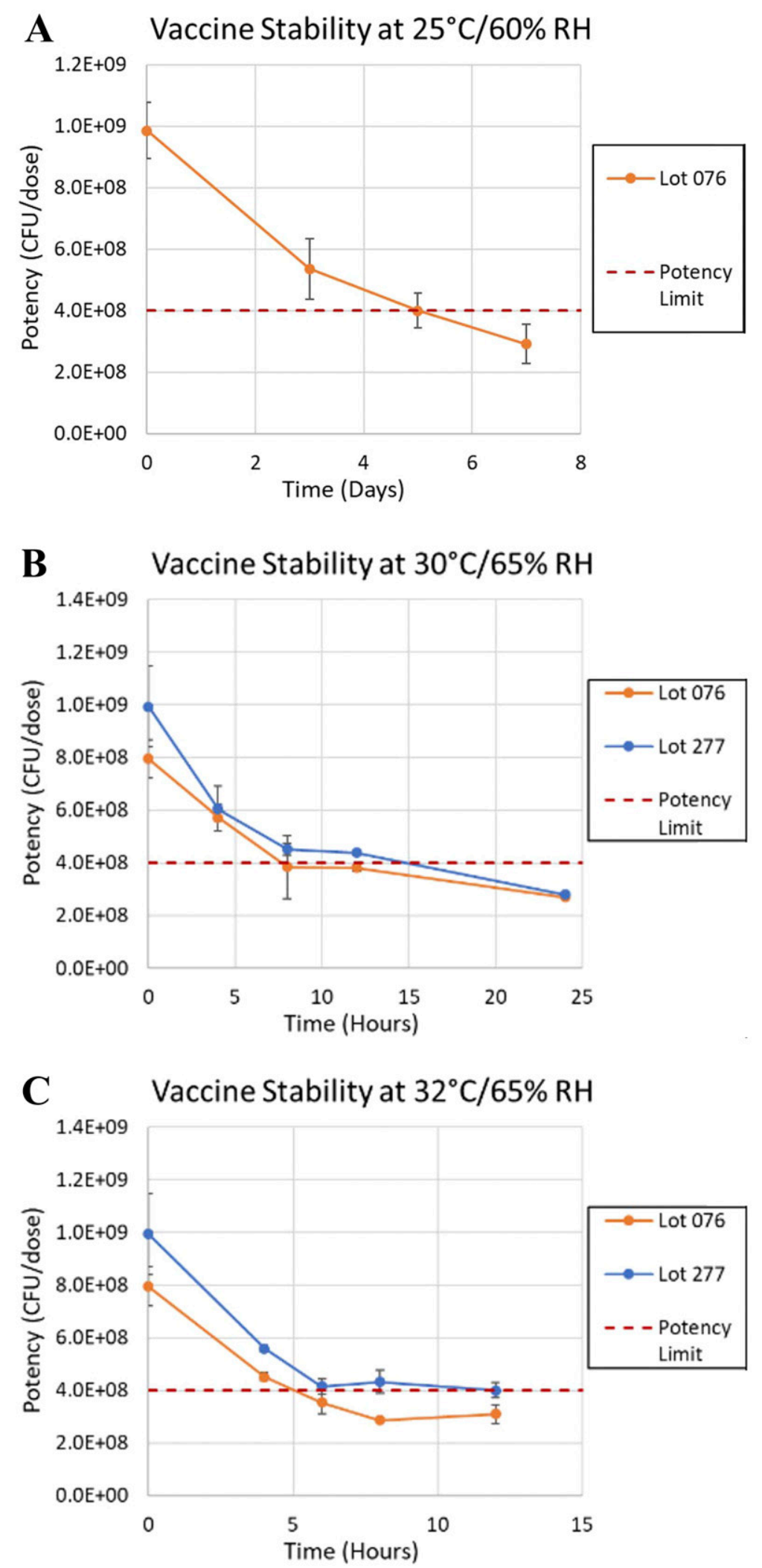

FIGURE 2. Stability of PXVX0200 vaccine at temperatures outside of the prescribed storage range. This figure appears in color at www.ajtmh.org. 
A 24 Hour Excursion Long-Term Stability (Lot 230)

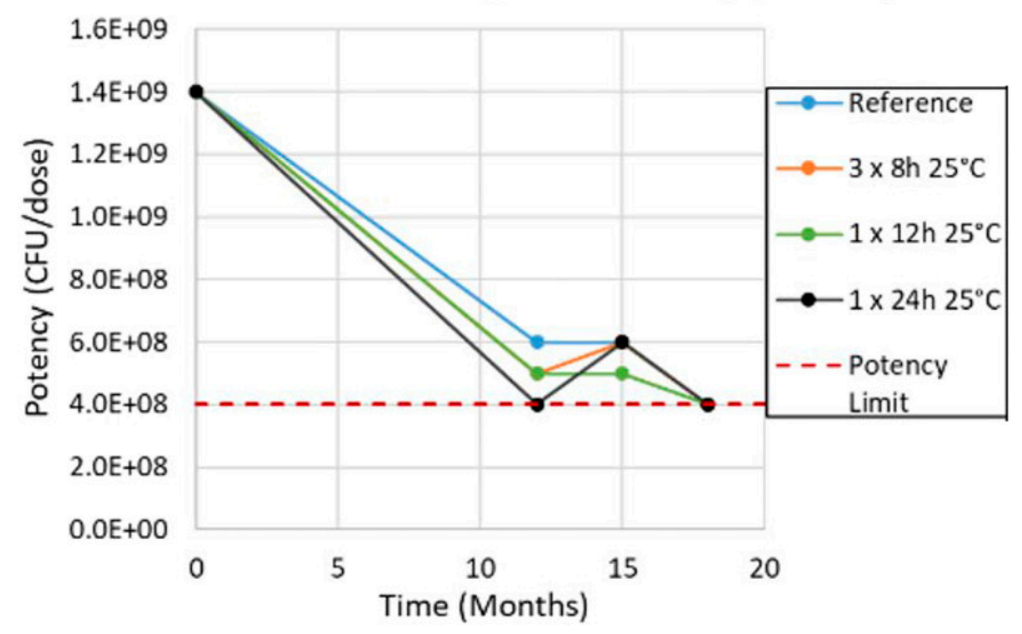

B 24 Hour Excursion Long-Term Stability (Lot 231)

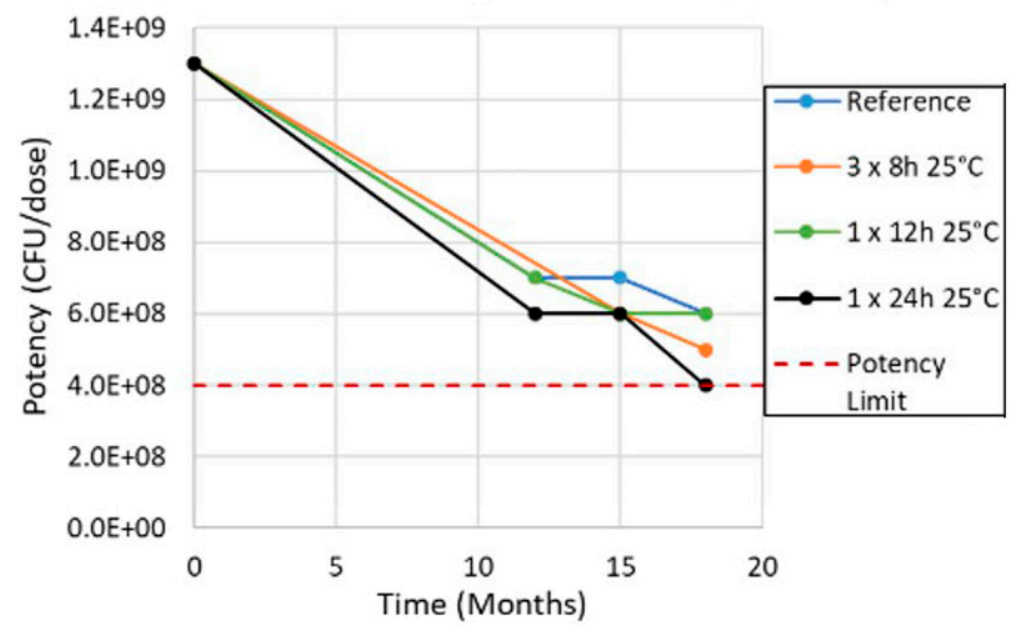

C 24 Hour Excursion Long-Term Stability (Lot 232)

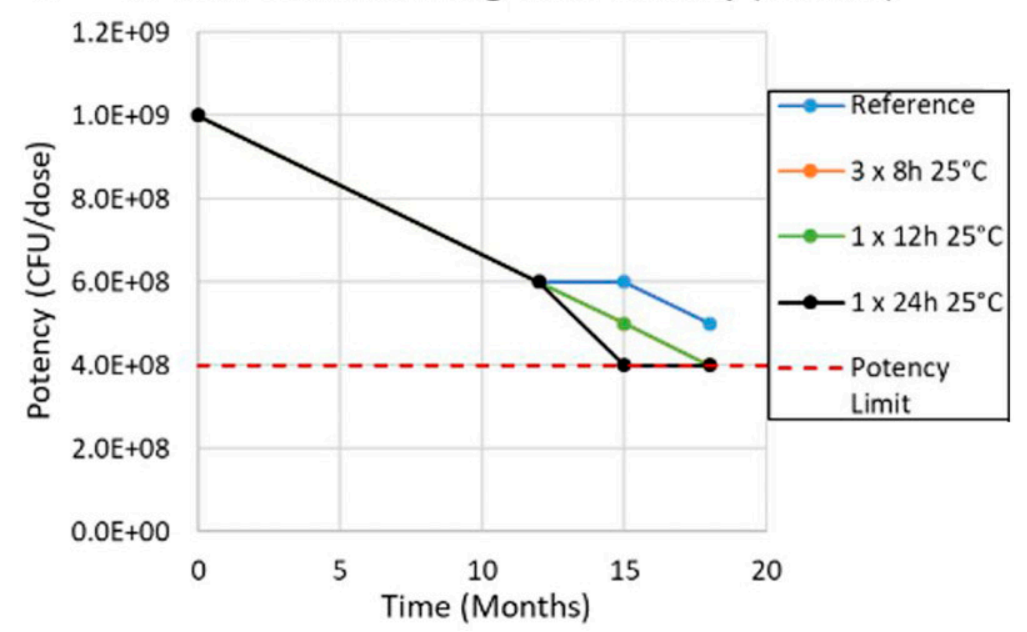

FIGURE 3. Long-term $2-8^{\circ} \mathrm{C}$ stability of vaccine following $25^{\circ} \mathrm{C} / 24$-hour excursions. This figure appears in color at www.ajtmh.org. 

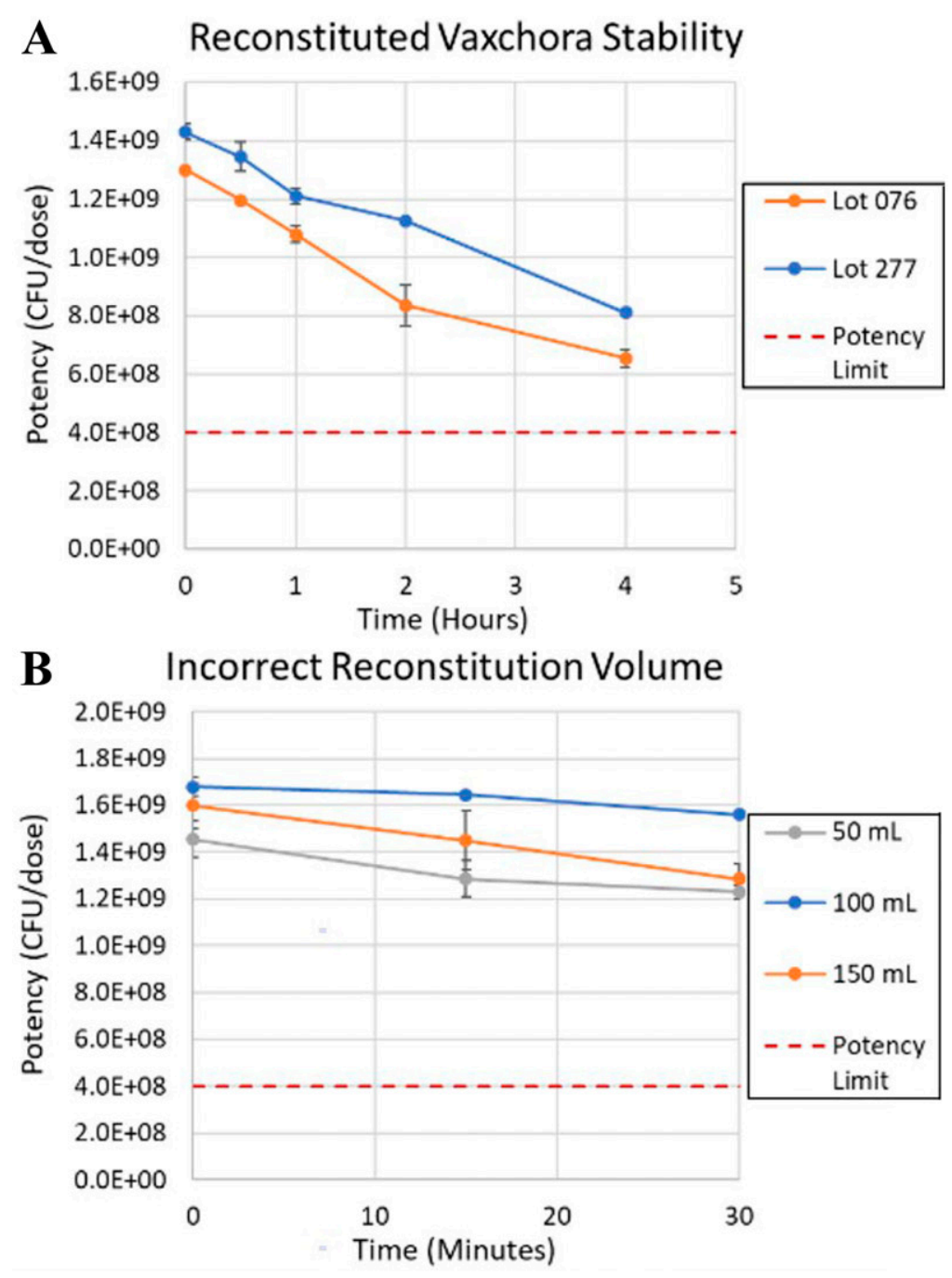

C Incorrect Packet Reconstitution Sequence

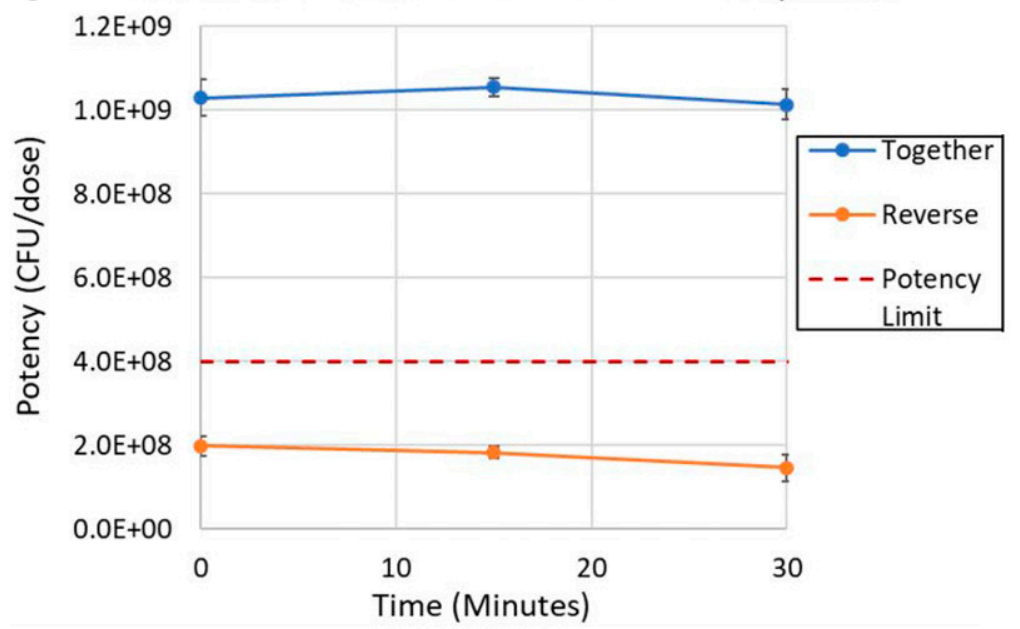

FIGURE 4. Stability of vaccine following misadministration errors. This figure appears in color at www.ajtmh.org. 
Samples taken for potency measurements immediately following reconstitution, and at 15 minutes and 30 minutes postreconstitution were diluted into chilled buffered peptone water and plated onto the TSA plates for analysis as described in Methods.

Packets reconstituted in reverse order. Vaccine potency was not retained when reconstitution of the buffer and vaccine component packet contents were conducted in reverse order, that is, vaccine component packet contents are added to $100 \mathrm{~mL}$ of water first followed by the buffer component packet contents.

Safe disposal of reconstituted PXVX0200 vaccine. The conditions tested for safe cleanup and disposal of PXVX0200 showed that hot water alone at $\leq 50^{\circ} \mathrm{C}$ would neutralize at most $88 \%$ of the CVD $103-\mathrm{HgR}$ bacteria, and that ambient water with the addition of antibacterial soap neutralizes $91 \%$ of the bacteria, when used as directed. Hot water $\left(50^{\circ} \mathrm{C}\right)$ with antibacterial soap neutralized $\geq 99 \%$ of the bacteria (Tables 1-3).

Hot water alone. Addition of hot water $\left(60^{\circ} \mathrm{C}\right)$ reduced vaccine titers to below detection limits (Table 1). Water at $40^{\circ} \mathrm{C}$ killed $43 \%$ of the CVD $103-\mathrm{HgR}$ V. cholerae, $50^{\circ} \mathrm{C}$ killed $88 \%$, and $60^{\circ} \mathrm{C}$ killed nearly $100 \%$ of the CVD $103-\mathrm{HgR}$ V. cholerae (99.7\%).

Soap and water. Sixty degree Celsius killed nearly $100 \%$, but safety guidance provided stipulates that water temperatures should not exceed $50^{\circ} \mathrm{C}$ to avoid scalding. This necessitated that studies be carried out using hot water and soap to neutralize active bacteria.

Neutralization experiments were conducted to determine effective conditions for decontamination. Antibacterial dish soap added to water at ambient temperature killed bacteria in a concentration dependent manner: $0.1 \mathrm{~mL}$ soap in $100 \mathrm{~mL}$ water killed $69 \%$ of the CVD 103-HgR V. cholerae and $5.0 \mathrm{~mL}$ soap in $100 \mathrm{~mL}$ of water killed $91 \%$ of the CVD 103-HgR V. cholerae (Table 2).

Antibacterial dish soap added to water at $50^{\circ} \mathrm{C}$ killed essentially all bacteria, irrespective of concentration of soap used: $0.1 \mathrm{~mL}$ soap in $100 \mathrm{~mL}$ water killed $99 \%$ of the CVD 103$\mathrm{HgR}$ V. cholerae and $5 \mathrm{~mL}$ soap in $100 \mathrm{~mL}$ of water killed $100 \%$ of the CVD 103-HgR V. cholerae (Table 3).

Therefore, both soap and hot water should be used to clean up spilled PXVX0200 vaccine material or residue left after administration.

\section{DISCUSSION}

Vaccine compliance is an important consideration during product development, especially for diseases such as cholera that are endemic in middle- to low-income countries. Travel vaccine requirements are rare, leaving the onus of compliance on travelers to both protect their own health and prevent the

TABLE 1

Hot water neutralization of Center for Vaccine Development 103-HgR Vibrio cholerae

\begin{tabular}{lccc}
\hline \multirow{2}{*}{$\begin{array}{c}\text { Water } \\
\text { temperature }\end{array}$} & $\begin{array}{c}\text { Potency } \\
\text { (colony-forming unit/dose) }\end{array}$ & 0 & 15 \\
\cline { 3 - 4 } $40^{\circ} \mathrm{C}$ & $1.2 \times 10^{9}$ & $6.9 \times 10^{8}$ & $6.0 \times 10^{8}$ \\
$50^{\circ} \mathrm{C}$ & $1.4 \times 10^{8}$ & $1.3 \times 10^{8}$ \\
$60^{\circ} \mathrm{C}$ & $3.3 \times 10^{6}$ & $\mathrm{BLQ}$ \\
\hline $\mathrm{BLQ}$ = below level of quantitation. & &
\end{tabular}

TABLE 2

Ambient temperature water and antibacterial soap neutralization of Center for Vaccine Development 103-HgR Vibrio cholerae

\begin{tabular}{lccccc}
\hline \multirow{2}{*}{$\begin{array}{c}\text { Soap } \\
\text { volume }\end{array}$} & $\begin{array}{c}\text { Potency } \\
\text { (colony-forming } \\
\text { unit/dose) }\end{array}$ & $\begin{array}{c}\text { Water } \\
\text { temperature }\end{array}$ & 0 & \multicolumn{3}{c}{ Post-reconstitution (minutes) } \\
\cline { 3 - 6 } & $1.4 \times 10^{9}$ & Ambient & $4.4 \times 10^{8}$ & $2.9 \times 10^{8}$ & $2.8 \times 10^{8}$ \\
$0.1 \mathrm{~mL}$ & $1.4 \times 10^{8}$ & $1.4 \times 10^{8}$ & $1.4 \times 10^{8}$ \\
\hline $\mathrm{mL}$ & & & $1.2 \times 10^{8}$ & $1.4 \times 1.4$ \\
\hline
\end{tabular}

spread of disease to vulnerable populations. The introduction and spread of disease in countries without the infrastructure to deal with an outbreak can be disastrous. PXVX0200 vaccine has characteristics that make it ideal as a vaccine to protect against $V$. cholerae, the causative agent in cholera disease. The vaccine is administered orally, requiring only a single dose to impart protection, key qualities that support vaccine recommendation compliance. As one of the final stages of development, studies were performed to characterize the vaccine to allow for self-administration in the comfort of one's own home.

PXVX0200 vaccine demonstrated both the stability and robustness to survive conditions that would be expected during the process of transporting and preparing the vaccine for self-administration. The vaccine was found to be stable outside of the prescribed refrigerated storage range for up to 5 days at $25^{\circ} \mathrm{C}$, and for several hours at temperatures up to $32^{\circ} \mathrm{C}$, which allows the patient to transport the vaccine home even during the hottest months of the year in Europe. Furthermore, vaccine retains long-term stability when it is taken out of the refrigerator for a short period of time and returned.

Once the vaccine has been transported home, we identified preparation steps in the PL where inadvertent mistakes, such as failing to consume the vaccine within 15 minutes following reconstitution, adding the vaccine and buffer packets together or in the wrong sequence, or using the incorrect reconstitution volumes, could result in misadministration of the vaccine. Risks were mitigated, where possible, with more specific instructions. Although PXVX0200 retained potency following reconstitution for at least 4 hours, on advice of the EMA, the revised packaged leaflet will adhere to current instructions to drink the reconstituted vaccine within 15 minutes as a safety measure to limit the risk of exposure of CVD 103-HgR to the environment. The vaccine retained potency when it was prepared incorrectly with the exception of reversed sequence addition of buffer and vaccine in which case potency requirements fell below specification. This led to the inclusion of a warning in the PL that PXVX0200 "may not work when mixing sachets in the wrong order; sachet 1 must be added to the water first. If the sachets are mixed in the wrong order you

TABLE 3

Hot water and antibacterial soap neutralization of Center for Vaccine Development 103-HgR Vibrio cholerae

\begin{tabular}{|c|c|c|c|c|}
\hline \multirow[b]{2}{*}{$\begin{array}{c}\text { Soap } \\
\text { volume }\end{array}$} & \multirow[b]{2}{*}{$\begin{array}{c}\text { Potency } \\
\text { (colony-forming unit/dose) }\end{array}$} & \multirow[b]{2}{*}{$\begin{array}{l}\text { Water } \\
\text { tempertaure }\end{array}$} & \multicolumn{2}{|c|}{ Post-reconstitution (minutes) } \\
\hline & & & 0 & 15 \\
\hline $\begin{array}{l}0.1 \mathrm{~mL} \\
5 \mathrm{~mL}\end{array}$ & $1.4 \times 10^{9}$ & $50^{\circ} \mathrm{C}$ & $\begin{array}{c}1.3 \times 10^{7} \\
B L Q\end{array}$ & $\begin{array}{c}1.9 \times 10^{6} \\
B L Q\end{array}$ \\
\hline
\end{tabular}


must discard the vaccine a request a replacement dose." (Supplement 1)

A method was determined for neutralizing the PXVX0200 active ingredient, CVD 103-HgR, using hot water and soap, in case of accidental spilled material or residue left over following administration. This led to the inclusion of instructions for the $\mathrm{PL}$ "if a spill occurs while stirring or drinking the medication, or there is any residue (powder or liquid left behind from the stirring utensil, cup, or other object) on the mixing surface, clean up spilled material or residue preferably with a disposable paper towel/cloth using hot water and soap or antibacterial disinfectant." This mitigates the risk of environmental impact from the GMO.

\section{CONCLUSION}

Taken together, these results give confidence that PXVX0200 vaccine is robust and, in addition to other attributes that support vaccine recommendation compliance, can be selfadministered, and disposed of safely.

Received September 17, 2020. Accepted for publication January 2, 2021.

Published online March 1, 2021.

Note: Supplemental materials and figure appear at www.ajtmh.org.

Authors' addresses: R. Paul Duffin, Michael Delbuono, and Amish A. Patel, Product Development, Emergent BioSolutions Inc, San Diego, CA, E-mails: duffinp@ebsi.com, michaeldelbuono@yahoo. com, and patela3@ebsi.com. Lawrence Chew, Analytical and Formulation Development, Emergent BioSolutions Inc, San Diego, CA, E-mail: chewl@ebsi.com. James Johnstone, Fermentation, Emergent BioSolutions Inc, San Diego, CA, E-mail: johnstonj@ebsi.com. Volker Niedan, Quality Control, Emergent BioSolutions Inc, San Diego, CA, E-mail: niedanv@ebsi.com. Pascal Schwarz, Manufacturing Operations, Emergent BioSolutions Inc, San Diego, CA, E-mail: schwarzp@ ebsi.com. Paul Shabram, Technical Operations, Emergent BioSolutions Inc, San Diego, CA, E-mail: shabramp@ebsi.com.

\section{REFERENCES}

1. Pietroni MAC, 2020. Case management of cholera. Vaccine 38 (Suppl 1): A105-A109.

2. Ali M, Nelson AR, Lopez AL, Sack DA, 2015. Updated global burden of cholera in endemic countries. PLoS Negl Trop Dis 9: e0003832.
3. Harvey K, Esposito DH, Han P, Kozarsky P, Freedman DO, Plier DA, Sotir MJ; CDC, 2013. Surveillance for travel-related disease-GeoSentinel surveillance system, United States, 1997-2011. MMWR Surveill Summ 62: 1-23.

4. Katz JM, 2016. U. N. Admits role in Cholera epidemic in Haiti. The New York Times. August 17, 2016.

5. Mavian C et al., 2020. Toxigenic Vibrio cholerae evolution and establishment of reservoirs in aquatic ecosystems. Proc Natl Acad Sci U S A 117: 7897-7904.

6. Azman AS, Luquero FJ, Ciglenecki I, Grais RF, Sack DA, Lessler J, 2015. The impact of a one-dose versus two-dose oral cholera vaccine regimen in outbreak settings: a modeling study. PLoS Med 12: e1001867.

7. Kristensen DD, Bartholomew K, Villadiego S, Lorenson K, 2016. What vaccine product attributes do immunization program stakeholders value? Results from interviews in six low- and middle-income countries. Vaccine 34: 6236-6242.

8. Ambrose CS, Wu X, 2013. The safety and effectiveness of selfadministration of intranasal live attenuated influenza vaccine in adults. Vaccine 31 : 857-860.

9. Norman JJ, Arya JM, McClain MA, Frew PM, Meltzer MI, Prausnitz MR, 2014. Microneedle patches: usability and acceptability for self-vaccination against influenza. Vaccine 32: 1856-1862.

10. White JA, Lal M, 2019. Technical product attributes in development of an oral enteric vaccine for infants. Vaccine 37 : 4800-4804.

11. Levine MM, Chen WH, Kaper JB, Lock M, Danzig L, Gurwith M, 2017. PaxVax CVD 103-HgR single-dose live oral cholera vaccine. Expert Rev Vaccines 16: 197-213.

12. Chen WH et al., 2016. Single-dose live oral cholera vaccine CVD 103-HgR protects against human experimental infection with Vibrio cholerae O1 El Tor. Clin Infect Dis 62: 1329-1335.

13. FDA, 2003. Guidance for Industry: Q1A(R2) Stability Testing of New Drug Substances and Products. www.fda.gov. Available at: https://www.fda.gov/media/71707/download. Accessed December 6, 2020.

14. EMA, 2003. ICH Topic Q 1 A (R2): Stability Testing of New Drug Substances and Products. Available at: https://www.ema. europa.eu/en/documents/scientific-guideline/ich-q-1-r2-stabilitytesting-new-drug-substances-products-step-5_en.pdf. Accessed December 6, 2020.

15. Current Results Publishing Ltd, 2020. Average July Temperatures for Cities in Europe 2020. Available at: https://www.currentresults.com/ Weather/Europe/Cities/temperature-july.php\#a. Accessed April 3, 2020.

16. Curtis V, Cairncross S, 2003. Effect of washing hands with soap on diarrhoea risk in the community: a systematic review. Lancet Infect Dis 3: 275-281.

17. The European Child Safety Alliance, 2010. Background to Position Statement: The Danger of Tap Water Scalds The European Child Safety Alliance. Available at: https://www.childsafetyeurope.org/ publications/positionstatements/info/tap-water-scaldsbackgrounder. pdf. Accessed April 3, 2020. 